\title{
Creating An Adolescent and Young Adult Cancer Program: Lessons Learned From Pediatric and Adult Oncology Practice Bases
}

\author{
Damon Reed, MDª Rebecca G. Block, $\mathrm{PhD}^{\mathrm{b}}$; and Rebecca Johnson, MDc
}

\begin{abstract}
Driven by reports of unmet clinical needs and lack of survival improvement, programs for adolescents and young adults (AYAs) with cancer have become increasingly common across the United States during the past 10 years. Programs generally originate from existing pediatric or adult hospitals, serve all or a subset of patients between 15 and 39 years of age at the time of cancer diagnosis, and aim to work collaboratively with other branches of their institution to deliver superior care for AYAs. Until recently, programs responded to local needs and evolved without an established framework for growth. Over the past several years, organizations including NCCN have published guidelines for AYA cancer care and for the development of clinical AYA programs. This article reviews these publications, describes the growth and development of 2 nationally recognized AYA centers-Seattle Children's Hospital and Moffitt Cancer Center-and offers practical suggestions to assist developing AYA programs. AYA oncology is entering a new era of increasing public recognition and nationally coordinated growth, as evidenced by the recent establishment of the Change it Back's Centers of Excellence Program that codifies criteria for excellence in AYA cancer care. AYA programs have the potential to improve care for a vital and underserved patient population, stimulate collaborative research, and enhance relationships with patients, the local community, referring physicians, and donors. (J Natl Compr Canc Netw 2014;12:1409-1415)
\end{abstract}

During the past 30 years, survival for many childhood cancers has dramatically increased due in part to brisk accrual onto multi-institutional clinical trials. ${ }^{1,2}$ Survival in older adults has also increased steadily because of im-

From aSarcoma Department, Moffitt Cancer Center, Tampa, Florida; ${ }^{b}$ Knight Cancer Institute, Oregon Health \& Science University, Portland, Oregon; and 'Seattle Children's Hospital/University of Washington, Seattle, Washington.

Submitted February 10, 2014; accepted for publication June 16, 2014.

The authors disclosed that they have no financial interests, arrangements, affiliations, or commercial interests with the manufacturers of any products discussed in this article or their competitors.

Correspondence: Damon Reed, MD, Sarcoma Department, Moffitt Cancer Center, 12902 Magnolia Drive, FOB 1, Tampa, FL 33612.

E-mail: Damon.Reed@moffitt.org provements in screening, increasingly effective therapies, and optimization of supportive care strategies. ${ }^{3,4}$ Cancer is the most common cause of disease-related death in adolescents and young adults (AYAs) aged 15 to 39 years, ${ }^{5}$ although this age group is the least studied age group. ${ }^{4} \mathrm{No}$ significant survival improvements have been realized in more than 3 decades, ${ }^{4}$ and AYAs have inferior outcomes in acute lymphoblastic leukemia (ALL), colon cancer, and breast cancer compared with other groups. ${ }^{2,6-9}$

Elucidating the reasons behind these disparities in outcome and survival improvement may reveal opportunities to improve cancer care for AYAs. Contributing factors occur throughout the cancer continuum. ${ }^{4,10-20}$ AYAs also have developmentally specific needs that must be addressed during cancer therapy, including fertility counseling, and they have distinctive social and emotional needs. ${ }^{410-20}$ Because most AYA cancer survivors will live for decades, coordinated transition to survivorship care is essential. ${ }^{13,16,21}$ An AYA program should be designed to address these diverse needs.

Multiple organizations worldwide have worked to characterize AYA cancer care as a specific discipline within oncology and to define the essential elements of care for this population. ${ }^{4,17,18,22}$ In 2012, the consensus-based NCCN Clinical Practice Guidelines in Oncology (NCCN Guidelines) for AYA Oncology were published..$^{23}$ Most recently, Change it Back created the Centers of Excellence Program to standardize and improve AYA care in US cancer centers, and defined 5 areas of service required for exemplary AYA cancer care: fertility counseling, health insurance and financial counseling, clinical trial education and facilitation, psychosocial support, and transition to survivorship services. ${ }^{21}$ The growth of clinical programs and guideline development has been an iterative process in the United States; the activities of existing AYA programs have been instrumental in informing each set of recommendations, guidelines, and criteria. This article describes the develop- 
ment of 2 AYA programs built as extensions of either medical oncology or pediatric oncology programs. It reviews key program elements based on both the experiences of the individual programs and the recommendations of published guidelines. ${ }^{13,17,18,21,22}$

\section{Pediatric-Based Program}

The AYA program at Seattle Children's Hospital (SCH) was founded by Dr. Rebecca Johnson in 2007 with the goal of expanding supportive services and clinical research, creating dedicated patient care facilities, and educating the community about AYA oncology. Program development during the first 2 years addressed the most glaring unmet needs of AYAs treated in a pediatric hospital. Institutional approval was obtained to provide multidisciplinary cancer care for patients up to 29 years of age at diagnosis, an "ideal state" model was developed for outpatient clinic flow (Figure 1), and an institution wide standard practice was created to ensure that all newly diagnosed male AYAs are offered sperm banking before the start of cancer therapy, which increased sperm banking attempts from $7 \%$ to $70 \%$ in the first year. ${ }^{24} \mathrm{~A}$ set of 10 concise educational handouts were written specifically targeting AYA patients with newly diagnosed cancer rather than their parents. ${ }^{25}$ The program team at $\mathrm{SCH}$ worked to transform the institutional culture to focus on the needs of AYAs by lecturing to nurses, physicians, nurse practitioners, and fellows, both in hematology/oncology and in many other divisions and departments. The team reached out to the psychology, adolescent medicine, urology, gynecology, physical therapy, and child life departments and teachers in the school at SCH, and to medical oncologists, nurse practitioners, and psychologists focusing on adult cancers at the University of Washington. These lectures and discussions enhanced the visibility of AYA oncology and identified champions for AYA cancer care within each specialty, rounding out the program's multidisciplinary team.

The team observed a serious lack of Internet video content on fertility preservation and the personal experiences of younger AYA patients with cancer. In response, the team created 2 sets of online videos that are now available on YouTube. One set focuses on AYA male ${ }^{26}$ and female fertility preservation. ${ }^{27}$ The second set of 12 videos, titled "Good Times and Bald Times," offers a firsthand glimpse into the experiences of AYA patients battling cancer and its aftermath. ${ }^{28}$ Unlike many videos on patients' cancer experiences, this series is searchable by topic (eg, hair loss, chemotherapy, relationships).

During the next several years, the team continued to tackle unmet needs and expand programming and infrastructure. The team now includes a medical director (Dr. Johnson), a clinical nurse specialist, a psychologist, a gynecologist embedded into the hematology/oncology clinic, a $\mathrm{PhD}$ researcher, and an AYA child life specialist, as well as AYA-focused nurses and ancillary staff supporting both patient care and research. An AYA patient advisory board reports on patient experiences and offers feedback on proposed projects. Supportive services aim to get patients out of bed to exercise, focus on life, and interact with other AYA patients. The program lends iPads, stationary bikes, yoga mats, pedometers, and interactive computer games that emphasize fitness. The team hosts weekly evening pizza and discussion sessions on the inpatient unit and supports an artist-in-residence focusing on AYAs with cancer. The program partners with numerous nonprofit organizations to promote and sponsor a variety of AYA-focused inpatient and outpatient activities, support groups, retreats, and camps; hosts a biennial patient education day; and YAC (Young Adult Cancer) Fest, Northwest" (www. yacfest.com) to boost awareness of the activities and services available in the geographic area.

The AYA research projects have been clinically focused, informing program development. The program has addressed unanswered questions regarding how to optimize medical and psychosocial services, and how to harness new communication technology in caring for AYAs with cancer. A patient assessment tool was created to stimulate age-appropriate discussion with providers and inform appropriate multidisciplinary referrals. The team has studied fertility preservation, the use of tablet computers to collect patient-reported outcome data from AYAs, and epidemiologic trends in AYA cancer incidence. . $4,29,30$ Ongoing studies address medication adherence, symptom reporting, emotional resilience, and spirituality in AYAs.

The team has emphasized community outreach to raise awareness of AYA cancer, speaking at local and regional community events for the general public and educational seminars for medical professionals. This work has led to rapid transformation of the program from a concept on paper into a national leader in AYA oncology research and clinical care. In early 


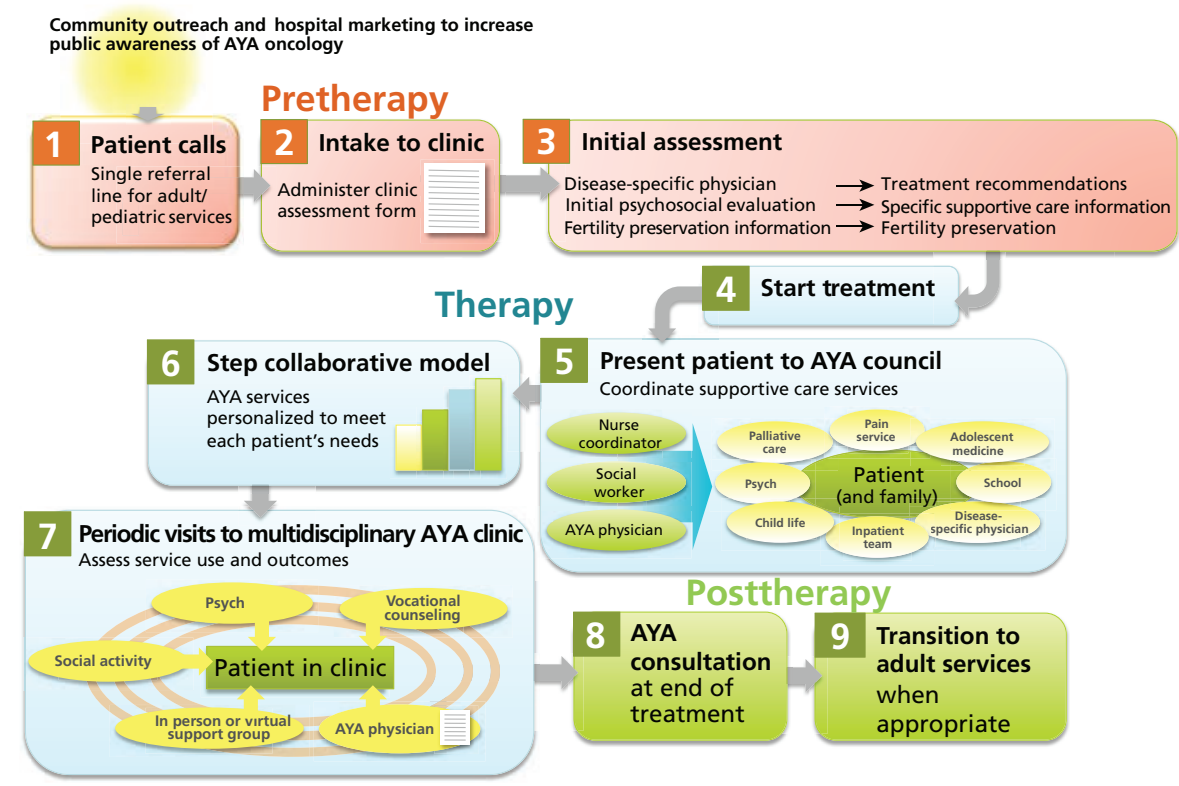

Figure 1 Seattle Children's Hospital ideal clinic flow model for adolescent and young adult (AYA) patients. Proactive institutional outreach improves regional awareness among referring physicians and the general public that AYAs with cancer may benefit from specialized services. At the time of initial referral, the institution should have a coordinated system through which newly diagnosed AYAs are triaged to the most appropriate care team within either pediatric or adult oncology services.

Courtesy of Dr. Rebecca Johnson.

2013, SCH opened a dedicated 16-bed inpatient AYA oncology unit comprising one-third of all oncology beds. AYAs at SCH now receive state-of-the-art medical and psychosocial care in age-appropriate facilities, delivered by a coordinated team of specialized staff.

Ongoing challenges include promoting appropriate referral patterns, overcoming the barrier of physical separation between pediatric and adult facilities, optimizing the use of the AYA patient assessment tool, and coordinating robust multidisciplinary service delivery within the existing clinic structure.

\section{Medical Oncology-Based Program}

Dr. Damon Reed works primarily at Moffitt Cancer Center (MCC), an adult NCCN Member Institution that does not routinely care for patients younger than 15 years. A senior physician, Dr. Douglas Letson, partnered with Dr. Reed to champion a proposal for organizing clinical services to meet the needs of AYA patients. The concept garnered widespread support from institutional leadership, clinical faculty, researchers, and nursing and social work staff and was supported by generous community donations. This level of support proved to be critical for the development of this program.
The team at MCC discovered that AYA patients aged 15 to 39 years constitute $12 \%$ of all new patients and total more than 2000 new patients per year; numbers that surprised and impressed the hospital administration. On a given day, MCC serves 100 to 150 AYA outpatients, and they occupy approximately $10 \%$ of inpatient beds. This number is relatively higher than the incidence of AYA cancer, suggesting that some AYA diagnoses require complex care that may generate revenue for the hospital.

The AYA program began within and was modeled on the MCC sarcoma clinic, a setting in which a significant percentage of patients are AYAs. ${ }^{31,32} \mathrm{New}$ AYA patients with sarcoma are seen within a week of appointment request, and the social worker, primary nurse, and pediatric oncologist evaluate the patient together. The process of assigning AYA patients to a pediatric oncologist was a local decision made by the chair; the model would also work well with a medical oncologist with AYA interests. The diagnosis is carefully explained, therapeutic options including clinical trials are presented, and risk to fertility is addressed. Patients interested in fertility preservation are seen by a nearby fertility clinic within 48 hours. For all new patients, scans, pathology, and the treatment plan are 
discussed at a biweekly multidisciplinary AYA tumor board or weekly sarcoma tumor board.

One of the team's first tasks was to solicit feedback and ideas from AYA patients themselves. They conducted a focus group in which newly diagnosed, on-therapy, and posttherapy patients spoke openly with little structure. The clear theme was that cancer was a lonely experience; participants voiced the desire for more peer interaction during and after therapy. Patients also wanted to learn more about risk of infertility and other late effects, improving relationships with their caregivers, and transitioning back to normal life after cancer therapy.

Next, the team formed an AYA committee at MCC that meets monthly with the goal of developing a comprehensive program to address the medical, psychosocial, reproductive, educational, and survivorship needs of this population. Current programming incudes social and educational events, monthly gatherings for AYA patients along with a support group for patients (Meet Up) and a parallel one for caregivers (Connect Up), and weekly art therapy for inpatients. An AYAspecific packet outlining the available internal and external resources is also provided to new patients.

Research focuses on many aspects of AYA care. The team has established rich interdisciplinary collaborations throughout MCC and obtained philanthropic support for a variety of projects. Among active projects, the team is collaborating with researchers to (1) sequence a subset of rare sarcomas, (2) compare clinical and molecular data from young and older patients to understand why AYA patients with colorectal carcinoma have inferior outcomes, and (3) survey reproductive choices of women of childbearing age who are survivors of meningioma.

The team believes the AYA program has infused the culture of the cancer center. Hospital leaders are translating their increasing knowledge about AYAs into new clinical programs. All new clinical staff are oriented on AYA issues, yearly mandatory educational assessments are collected, and AYA topics are stressed during daily rounds.

Ongoing challenges include engaging patients to consistently attend events and interact with the staff. The program is enhancing its social media presence and creating a physical space for AYAs to improve its interface with patients.

\section{Essential Elements of a Program}

Based on experience in AYA program development and other organizations, including NCCN, Teenage Cancer Trust, the LIVESTRONG Young Adult Alliance, and Change it Back, the authors offer a synthesized conceptualization of the elements of an AYA program. ${ }^{4,13,18,21}$ The elements described may inform the development of new clinical programs and clarify AYA programs as a care delivery concept. Table 1 suggests first steps for programs in the initial phases of starting an AYA program.

The goal of AYA clinical programs is to deliver family-centered care with the patient as the focus, modeling the strengths of pediatrics and medical oncology, respectively. ${ }^{33}$ AYAs should have access to health care professionals with disease-specific expertise. Treatment should be provided with cure as the goal when possible, recognizing that AYAs can tolerate intensive therapy. ${ }^{4,13,18}$ In addition to standard symptom management, age-appropriate supportive care for AYAs includes timely fertility counseling and referral, and counseling to address sexual dysfunction and the negative effects of treatment on body image. ${ }^{4,17,34}$ Issues of substance use, contraception, and sexually transmitted infection prevention should also be addressed consistently. ${ }^{17}$ Providers should address adherence, which may be improved by good communication and a positive therapeutic alliance. ${ }^{17,35}$ Treatment planning should always involve consideration of late effects, given AYAs' longer life expectancy compared with older adults..$^{13,21}$

Psychosocial care should include comprehensive assessment at diagnosis, with ongoing monitoring through treatment and survivorship. Areas of inquiry include mood, coping, healthful behaviors, substance use, social support, and family, work, school, and home environment. ${ }^{13,17,18}$ Developmentally appropriate psychosocial interventions should be available to AYAs based on the results of their assessments. Interventions may range from mental health referrals to support services, such as psychoeducational groups and vocational counseling. Information and advocacy about insurance and financial assistance are important to AYA care, as is patient education. ${ }^{13,17,18}$ Educational materials should be developmentally appropriate in content and design, and address both medical and psychosocial topics. AYA patients should be the focus of the teaching, with active participation from the 


\begin{tabular}{|c|c|c|}
\hline Role & & ggested Tasks \\
\hline Medical champion & $\begin{array}{l}\square \\
\square \\
\square\end{array}$ & $\begin{array}{l}\text { Define providers responsible for AYA cancers and facilitate collaboration between pediatric and adult services. } \\
\text { Set up regular (perhaps quarterly) tumor board-type meetings in which medical and pediatric oncologists jointly } \\
\text { discuss patient cases. Consider starting with specialists in disease groups that see many AYAs. Raise awareness and } \\
\text { build knowledge across administration, clinical, research, nursing, social work, staff. Give presentations about AYA } \\
\text { oncology-specific issues within the hospital and the community. } \\
\text { Become familiar with Change it Back's Center of Excellence criteria and consider working toward certification. } \\
\text { Participate in AYA-specific regional national meetings, such as Critical Mass, Society for Adolescent and Young } \\
\text { Adult Oncology, and the annual Texas Adolescent \& Young Adult Oncology Conference. } \\
\text { Emphasize and investigate opportunities for AYA research. Identify research projects and collaborations within } \\
\text { and across institutions. Encourage AYA participation in clinical trials. } \\
\text { Build and lead a multidisciplinary team with a clear mission statement to improve care of AYA patients. }\end{array}$ \\
\hline $\begin{array}{l}\text { Program } \\
\text { coordinator (with } \\
\text { medical champion) }\end{array}$ & $\begin{array}{l}\square \\
\square \\
\square\end{array}$ & $\begin{array}{l}\text { Define which providers will offer fertility preservation at diagnosis, and create a system to facilitate and track } \\
\text { referrals, and assess on an ongoing basis. } \\
\text { Coordinate the multidisciplinary AYA team: } \\
\square \text { Develop roles to build on members' strengths } \\
\square \text { Create and implement schedule for regular meetings } \\
\square \text { Develop a mechanism for contacting all members } \\
\text { Create timeline and present vision and resources needed, including full-time equivalents (liaisons, programming), } \\
\text { events, physical space, infrastructure, and education. Contact local nonprofit groups and explore collaborations } \\
\text { and/or grants. }\end{array}$ \\
\hline $\begin{array}{l}\text { Administrative } \\
\text { assistant (with } \\
\text { program } \\
\text { coordinator) }\end{array}$ & $\begin{array}{l}\square \\
\square \\
\square \\
\square\end{array}$ & $\begin{array}{l}\text { Create a system for contacting patients for social events. } \\
\text { Coordinate some social events for patients; initial events might include focus groups for baseline assessment of } \\
\text { patient needs and care preferences. } \\
\text { Create a patient advisory board to continuously inform program development. } \\
\text { Create and use social media platforms to communicate program events and information about AYA cancer. }\end{array}$ \\
\hline $\begin{array}{l}\text { Oncology clinic } \\
\text { director (with } \\
\text { medical champion) }\end{array}$ & & $\begin{array}{l}\text { lore models for: } \\
\square \quad \text { Providing outpatient care to AYAs } \\
\square \quad \text { Fertility experts (navigator, clinic, researchers, quality) } \\
\square \text { Psychosocial assessment and survivorship education } \\
\square \text { Clinic flow for provision of services by multidisciplinary team } \\
\square \text { Clustering inpatient care for AYAs }\end{array}$ \\
\hline $\begin{array}{l}\text { Hospital } \\
\text { administrator (with } \\
\text { medical champion) }\end{array}$ & $\begin{array}{l}\square \\
\square \\
\square\end{array}$ & $\begin{array}{l}\text { Define age range for AYA program. } \\
\square \text { Create system to ensure that all patients in AYA age range will be cared for by the hospital's subspecialists and } \\
\text { other staff } \\
\text { Discuss possibilities and timeline for creating dedicated space for inpatient and outpatient care of AYAs with } \\
\text { cancer. } \\
\text { Obtain ongoing funding for AYA program through local philanthropic support and/or nonprofit organizations, } \\
\text { such as Teen Cancer America (for patients } 14-24 \text { years of age). }\end{array}$ \\
\hline
\end{tabular}

Abbreviation: AYA, adolescent and young adult.

caregivers, family, and friends selected by the patient. ${ }^{33}$ The influence of treatment setting and its potential to contribute to or detract from quality AYA care should not be underestimated. Clustering inpatient and outpatient care creates opportunities for patients to meet and interact. ${ }^{17,18}$ Inpatient care is made AYA-friendly by making routines and visiting policies flexible and age-appropriate, minimizing time in the hospital, and creating clear ground rules for conduct on the unit. Providing Internet access and encouraging patients to personalize their hospital environment can add comfort to inpatient stays. ${ }^{17}$ Group activities and dedicated common space with developmentally appropriate décor, mu- sic, and games encourage patient interactions and minimize the isolation many AYAs experience. ${ }^{18,22}$

Outpatient care can be made AYA-specific by providing a common space for AYAs to gather, offering support groups for these patients, and hosting social activities. Groups and activities should be free of charge, include assistance with transportation and childcare, and allow survivors to bring a friend or sibling. Social networking is a necessary and useful tool in publicizing groups and activities. ${ }^{13,17,18,22}$

AYA-specific care should be delivered by a multidisciplinary team with specialized skills. ${ }^{18,22}$ Specific institutional approaches may vary based on local needs and available services. ${ }^{17}$ Table 2 lists the most 
common elements of an AYA multidisciplinary team in the United States according to a recent national survey (Rebecca Block, $\mathrm{PhD}$, personal communication). A multidisciplinary team may include AYAfocused professionals and paraprofessionals, including physicians, nurses, and representatives from patient navigation (team coordinator or patient educator/ navigator), educational/vocational support, genetic services, survivorship services, palliative care, and pastoral care, and administrative support. The team meets regularly to discuss AYAs treated at the institution, with each case considered from a broad range of viewpoints and potential interventions. ${ }^{18,22}$

All AYA team members should be educated on the cognitive, psychiatric, and psychosocial needs of AYA patients. Compared with older adults, AYAs benefit from more time with health care professionals and staff, which is key to a strong therapeutic alliance. ${ }^{17,34,35}$ AYAs may also require significant advocacy from providers, and encouragement to recognize limitations, care for themselves, and ask for help. ${ }^{17,18}$ Staff must maintain confidentiality with patients and recognize that those older than 18 years can choose their caregivers. Countertransference and boundaries can be challenged in AYA care, because many staff are in the same age range as patients. ${ }^{17}$ Multiple resources exist for training and continuing education in AYA care, including the Nurse Oncology Education Program, "At the Crossroads: Cancer in Ages 15-39" ${ }^{6}$ and the ASCO "Focus Under Forty" educational series for physicians. ${ }^{37}$

Research especially toward successful interventions is essential within AYA programs given the inferior survival improvements and documented unmet psychosocial needs of this population. ${ }^{4,16,38}$ Significant knowledge gaps exist within the areas of therapeutics, supportive care, outcomes, psychosocial adjustment, and basic research. ${ }^{4}$ Increasing clinical trial accrual is critical to improving AYA cancer survival. ${ }^{7}$ At one center, clinical focus on promoting enrollment on therapeutic trials significantly increased accrual..$^{39}$ Optimally, new therapeutic trials involving common AYA cancers should include the entire 15 - to 39 -year-old age spectrum..$^{40} \mathrm{New}$ and ongoing quality-of-life studies that evaluate coping, care models, fertility/sexuality, education or career re-entry, advance care directives, and survivorship will enhance existing knowledge and improve AYA care worldwide. Basic science research explores top-

\begin{tabular}{|c|c|}
\hline Service or Provider & $\begin{array}{l}\text { No. of AYA } \\
\text { Programs (\%) }\end{array}$ \\
\hline AYA champion & $18(90 \%)$ \\
\hline $\begin{array}{l}\text { Community-based patient service } \\
\text { organizations }\end{array}$ & $17(85 \%)$ \\
\hline Pediatric oncologist & $16(80 \%)$ \\
\hline Oncology nurse & $13(65 \%)$ \\
\hline Mental health professionals & $13(65 \%)$ \\
\hline Peer support & $13(65 \%)$ \\
\hline Child life specialist & $8(40 \%)$ \\
\hline $\begin{array}{l}\text { Reproductive endocrinologist/fertility } \\
\text { specialist }\end{array}$ & $4(20 \%)$ \\
\hline Oncology specialists & $3(15 \%)$ \\
\hline $\begin{array}{l}\text { Vocational rehabilitation/occupational } \\
\text { therapist }\end{array}$ & $3(15 \%)$ \\
\hline Patient/peer navigator & $2(10 \%)$ \\
\hline Palliative care specialists & $2(10 \%)$ \\
\hline Physical therapist, fitness trainer, coach & $2(10 \%)$ \\
\hline Geneticist/genetic counselor & $2(10 \%)$ \\
\hline Nutritionist/dietitian & $1(5 \%)$ \\
\hline AYA-trained hospice team & $1(5 \%)$ \\
\hline Primary care provider & $0(0 \%)$ \\
\hline Religious/spiritual counsel/chaplaincy & $0(0 \%)$ \\
\hline Legal counsel & $0(0 \%)$ \\
\hline Sexual health specialist & $0(0 \%)$ \\
\hline
\end{tabular}

Abbreviation: AYA, adolescent and young adult.

ics such as how reported cancer incidence and/or outcome differences in AYAs relate to fundamental age-related differences in tumor biology. ${ }^{1,6-8,30,41-43}$

Community outreach focuses on increasing the visibility of the AYA program within the cancer center and the community, with the goals of sharing knowledge, raising funds for clinical programming and research, and increasing referrals. Outreach activities target oncologists, primary care providers, and the general community, and may include educational sessions to raise awareness of AYA issues, and social media outreach such as Facebook postings, tweets, and videos created by AYA patients and staff. ${ }^{40}$

\section{Conclusions}

The number of specific programs for AYAs with cancer is increasing at a rapid rate throughout the United States. Previously offered at only a few major referral centers, AYA programs are now advertised by an increasing number of academic centers, community hospitals, and hospital systems. AYA patients constitute a significant volume of patients and thus represent a potential source of revenue for both pe- 
diatric and adult medical institutions. Research in AYA oncology bears excellent potential for funding from sources that emphasize interdisciplinary collaboration. ${ }^{40}$ Clustering care for AYAs within designated programs adhering to nationally accepted standards will result in improved medical and psychosocial outcomes for this important population.

\section{Acknowledgments}

For the Moffitt AYA Program: Research is supported by the generosity of the Gonzmart family foundation (www. richardsrunforlife.org/our-cause.html) and program development by Swim Across America, Tampa (www.swimacrossamerica.org/site/PageServer?pagename=TAM_ Home). Dr. Reed also receives support from The Pediatric Cancer Foundation (http://fastercure.org). The authors wish to thank LIVESTRONG for supporting the research on AYA programs and Karen Albritton and Brandon Hayes-Lattin.

\section{References}

1. Mullighan CG, Willman CL. Advances in the biology of acute lymphoblastic leukemia-from genomics to the clinic. J Adolesc Young Adult Oncol 2011;1:77-86.

2. Schafer ES, Hunger SP. Optimal therapy for acute lymphoblastic leukemia in adolescents and young adults. Nat Rev Clin Oncol 2011;8:417-424.

3. Edwards BK, Noone AM, Mariotto AB, et al. Annual report to the nation on the status of cancer, 1975-2010, featuring prevalence of comorbidity and impact on survival among persons with lung, colorectal, breast, or prostate cancer. Cancer 2014;120:1290-1314.

4. Adolescent and Young Adult Oncology Progress Review Group. Closing the gap: research and care imperatives for adolescents and young adults with cancer Available at: http://planning.cancer.gov/library/AYAO_PRG_Report_2006_ FINAL.pdf. Accessed September 3, 2014.

5. Bleyer A, Barr R. Introduction - impact of malignant diseases on young adults II. Semin Oncol 2009;36:380.

6. Bleyer A, Barr R, Hayes-Lattin B, et al. The distinctive biology of cancer in adolescents and young adults. Nat Rev Cancer 2008;8:288-298.

7. Keegan TH, Press DJ, Tao L, et al. Impact of breast cancer subtypes on 3-year survival among adolescent and young adult women. Breast Cancer Res 2013;15:R95.

8. Tricoli JV, Seibel NL, Blair DG, et al. Unique characteristics of adolescent and young adult acute lymphoblastic leukemia, breast cancer, and colon cancer. J Natl Cancer Inst 2011;103:628-635.

9. Zbuk K, Sidebotham EL, Bleyer A, La Quaglia MP. Colorectal cancer in young adults. Semin Oncol 2009;36:439-450.

10. Albritton KH, Eden T. Access to care. Pediatr Blood Cancer 2008;50(5 Suppl):1094-1098.

11. Arnett JJ, Tanner JL, eds. Emerging Adults in America: Coming of Age in the 21st Century. 1st ed. Washington, DC: American Psychological Association; 2006.

12. Carey ML, Clinton-McHarg T, Sanson-Fisher RW, Shakeshaft A. Development of cancer needs questionnaire for parents and carers of adolescents and young adults with cancer. Support Care Cancer 2012;20:991-1010.

13. Coccia PF, Pappo AS, Altman J, et al. NCCN Guidelines Insights: Adolescent and Young Adult Oncology, Version 2.2014. J Natl Compr Canc Netw 2014;12:21-32. To view the complete and most recent version of these guidelines, visit NCCN.org.

14. Erikson EH. Identity, Youth, and Crisis. 1st ed. New York, NY: Norton; 1968.

15. Keegan TH, Lichtensztajn DY, Kato I, et al. Unmet adolescent and young adult cancer survivors information and service needs: a population-based cancer registry study. J Cancer Surviv 2012;6:239-250.
16. Smith AW, Parsons HM, Kent EE, et al. Unmet support service needs and health-related quality of life among adolescents and young adults with cancer: the AYA HOPE study. Front Oncol 2013;3:75.

17. Smith S, Case L, Waterhouse $K$, et al. A Blueprint of Care for Teenagers and Young Adults with Cancer. Available at: http://www.teenagecancertrust.org/ workspace/documents/Blueprint-of-care.pdf. Accessed January 23, 2014.

18. Zebrack B, Mathews-Bradshaw B, Siegel S. Quality cancer care for adolescents and young adults: a position statement. J Clin Oncol 2010;28:4862-4867.

19. Clinton-McHarg T, Carey M, Sanson-Fisher R, et al. Preliminary development and psychometric evaluation of an unmet needs measure for adolescents and young adults with cancer: the Cancer Needs Questionnaire - Young People (CNQ-YP). Health Qual Life Outcomes 2012;10:13.

20. Burke ME, Albritton K, Marina N. Challenges in the recruitment of adolescents and young adults to cancer clinical trials. Cancer 2007;110:2385-2393.

21. Change it Back: AYA Cancer Alliance. Health Care Rights Initiative Web site. available at: http://hcri.org/programs/change-it-back/. Accessed September 3, 2014.

22. Ferrari A, Thomas D, Franklin AR, et al. Starting an adolescent and young adult program: some success stories and some obstacles to overcome. J Clin Oncol 2010;28:4850-4857.

23. Coccia PF, Altman J, Bhatia S, et al. NCCN Clinical Practice Guidelines in Oncology: Adolescent and Young Adult Oncology. J Natl Compr Canc Netw 2012;10:1112-1150. To view the most recent version of these guidelines, visit NCCN.org.

24. Shnorhavorian M, Kroon L, Jeffries H, Johnson R. Creating a standardized process to offer the standard of care: continuous process improvement methodology is associated with increased rates of sperm cryopreservation among adolescent and young adult males with cancer. J Pediatr Hematol Oncol 2012;34:e315-319.

25. Adolescent and Young Adult (AYA) Cancer Program. Seattle Children's Hospital Web site. Available at: http://www.seattlechildrens.org/clinicsprograms/cancer/services/adolescent-young-adult-program/. Accessed January 30,2014 .

26. Seattle Children's - Cancer Treatments and Fertility - Your Options (for males) [Video]. YouTube. Available at: http://www.youtube.com/watch?v=MppbxrBqA8\&feature=youtu.be. Published September 26, 2013. Accessed September 3, 2014.

27. Cancer Treatments and a Woman's Fertility [Video]. YouTube. Available at: https://www.youtube.com/watch?v=aySswe8NMgI. Published November 3, 2010. Accessed September 3, 2014.

28. SeattleChildrens. Teens and Young Adults With Cancer [Playlist]. YouTube. Available at: https://www.youtube.com/playlist?list=PLFOEBE7A44B2A27BC. Accessed September 3, 2014.

29. Wu WW, Johnson R, Schepp KG, Berry DL. Electronic self-report symptom and quality of life for adolescent patients with cancer: a feasibility study. Cancer Nurs 2011;34:479-486.

30. Johnson RH, Chien FL, Bleyer A. Incidence of breast cancer with distant involvement among women in the United States, 1976 to 2009. JAMA 2013;309:800-805.

31. Amankwah EK, Conley AP, Reed DR. Epidemiology and therapies for metastatic sarcoma. Clin Epidemiol 2013;5:147-162.

32. Burningham Z, Hashibe M, Spector L, Schiffman JD. The epidemiology of sarcoma. Clin Sarcoma Res 2012;2:14

33. Bleyer AW, Barr RD, eds. Cancer in Adolescents and Young Adults. New York, NY: Springer; 2007.

34. Zebrack $B$, Isaacson $S$. Psychosocial care of adolescent and young adult patients with cancer and survivors. J Clin Oncol 2012;30:1221-1226.

35. Johnson RH. TOUCH of the healer: defining core behaviors of oncologists that affect the mental health of cancer patients. Cancer 2014;120:2233-2236.

36. At the Crossroads: Cancer in Ages 15-39. Nurse Oncology Education Program. Available at: http://www.noep.org/nursing-cne/preview/40-at-the-crossroadscancer-in-ages-15-39. Accessed September 3, 2014.

37. Focus Under Forty. ASCO University Web site. Available at: http://university. asco.org/focus-under-forty. Accessed September 3, 2014

38. Zebrack BJ, Block R, Hayes-Lattin B, et al. Psychosocial service use and unmet need among recently diagnosed adolescent and young adult cancer patients. Cancer 2013;119:201-214

39. Shaw $\mathrm{PH}$, Boyiadzis $\mathrm{M}$, Tawbi $\mathrm{H}$, et al. Improved clinical trial enrollment in adolescent and young adult (AYA) oncology patients after the establishment of an AYA oncology program uniting pediatric and medical oncology divisions. Cancer 2012;118:3614-3617.

40. Johnson RH. AYA in the USA. International perspectives on AYAO, part 5. J Adolesc Young Adult Oncol 2013;2:167-174.

41. Wood WA, Lee SJ. Malignant hematologic diseases in adolescents and young adults. Blood 2011;117:5803-5815.

42. Gramatges MM, Rabin KR. The adolescent and young adult with cancer: state of the art-- acute leukemias. Curr Oncol Rep 2013;15:317-324.

43. Hubbard JM, Grothey A. Adolescent and young adult colorectal cancer. J Natl Compr Canc Netw 2013;11:1219-1225. 